http://dx.doi.org/10.35381/racji.v5i1.622

\title{
El principio de proporcionalidad de las penas en delitos cometidos por menores infractores
}

\section{The Proportionality Principles of penalties for crimes committed by juvenile offenders}

\author{
Rosa Maricela Peñaloza-Caracundo \\ rosa.penaloza@psg.ucacue.edu.ec \\ Universidad Católica de Cuenca, Cuenca \\ Ecuador \\ https://orcd.org/0000-0002-7098-9788 \\ Cecilia Ivonne Narváez-Zurita \\ inarvaez@ucacue.edu.ec \\ Universidad Católica de Cuenca, Cuenca \\ Ecuador \\ https://orcid.org/0000-0002-7437-9880 \\ Enrique Eugenio Pozo-Cabrera \\ epozo@ucacue.edu.ec \\ Universidad Católica de Cuenca, Cuenca \\ Ecuador \\ https://orcid.org/0000-0003-4980-6403 \\ Juan Carlos Erazo-Álvarez \\ jcerazo@ucacue.edu.ec \\ Universidad Católica de Cuenca, Cuenca \\ Ecuador \\ https://orcid.org/0000-0001-6480-2270
}

Recibido: 15 de noviembre de 2019

Aprobado: 16 de diciembre de 2019 


\title{
RESUMEN
}

La Constitución de la República de Ecuador, en su articulado señala de forma concreta derechos, garantías, deberes y principios constitucionales de sus ciudadanos. Uno de sus principios que se encuentran en nuestra constitución es la proporcionalidad. Por lo tanto, el objetivo de esta investigación consiste en analizar y determinar la efectiva aplicación del principio de proporcionalidad de las penas en los delitos cometidos por menores o adolescentes infractores sustentada en las garantías del debido proceso. La metodología utilizada en la investigación es de modalidad Cualitativa de análisis crítico, de tipo explicativa, siguiendo los métodos Inductivo-Deductivo y Analítico-Sintético, de alcance exploratorio; revisión bibliográfica sustentada en el análisis, síntesis y esquematización de la información. Al finalizar la investigación ha sido posible establecer que es una realidad el incumplimiento del principio de proporcionalidad en la determinación de la pena en los casos de delitos cometidos por menores o adolescentes infractores.

Descriptores: Principio de proporcionalidad; menores infractores; derecho constitucional; debido proceso; responsabilidad penal.

\begin{abstract}
The Constitution of the Republic of Ecuador, in its article specifically indicates the rights, guarantees, duties and constitutional principles of its citizens. One of its principles found in our constitution is proportionality. Therefore, the objective of this investigation is to analyze and determine the effective application of the principle of proportionality of penalties in crimes committed by minors or teenagers who are offenders based on the guarantees of due process. The methodology used in the research is of Qualitative modality of critical analysis, of explanatory type, following the Inductive-Deductive and Analytical-Synthetic methods, of exploratory scope; literature review based on the analysis, synthesis and schematization of information. At the end of the investigation it has been possible to establish that a breach of the principle of proportionality in the determination of the penalty is a reality in the cases of crimes committed by minors or teenagers who are offenders.
\end{abstract}

Descriptors: Principle of proportionality; juvenile offenders; constitutional right; due process; criminal liability 


\section{INTRODUCCIÓN}

El Derecho Constitucional es considerado en la actualidad una rama del Derecho Público, encargado de realizar el análisis de las normas que hacen alusión a la organización o estructura de los gobiernos, la competencia y atribuciones de cada una de las funciones del Estado; y, principalmente, determinar los derechos, garantías y obligaciones de los ciudadanos de forma individual, especificando las instituciones estatales encargadas de otorgar seguridad sobre su carácter protectivo (Urzua, 1991). Es así como la Constitución de la República del Ecuador, aprobada en el mes de octubre del año 2008, es garantista y por consiguiente defiende el fiel cumplimiento de los derechos, aunque en materia de obligaciones contiene mínimas y específicas estipulaciones, contenidas sobre todo en el Art. 83 del cuerpo legal.

De acuerdo al sentido garantista de la Constitución del Ecuador, los adolescentes constituyen un sector de atención prioritaria y sus derechos se especifican dentro del capítulo tercero que habla precisamente de las personas y grupos de atención prioritaria. En este sentido, el Art. 44 de la mencionada norma jurídica, establece que es obligación del Estado ecuatoriano "brindar protección, apoyo y promover el desarrollo integral, de Niños Niñas y Adolescentes" (Asamblea Nacional, 2008, p. 34). La garantía se hará efectiva durante todo el proceso del desarrollo de los menores, fomentando la educación, la salud, el acceso a derechos de bienestar familiar, social, etc. Los adolescentes gozan de la igualdad de derechos que en forma general se otorgan a la sociedad, constando eso en el Art. 45 de la misma Constitución.

Al igual que el Estado otorga garantía constitucional a los derechos que gozan los adolescentes menores de edad, hay que tener en cuenta sus deberes y obligaciones, que tal como señala el Código Orgánico de la Niñez y Adolescencia del Ecuador (2012), artículo 64, inciso primero "se imponen a los ciudadanos en cuanto sean compatibles con su condición y etapa evolutiva" (p. 12). Son deberes concretos y específicos de los menores de edad respetar los derechos de las demás personas y cultivar este valor, así como los de justicia y paz entre otros; dando prioridad a cumplir con su período de 
estudio, honras y respetar a sus padres; deberes que en conjunto con los especificados en este código y los consagrados en el Art. 83 de la Carta Magna, harán que los menores de edad y adolescentes se formen como ciudadanos de bien.

Desde hace algunos años los menores han venido siendo utilizados como instrumentos para el cometimiento de delitos por parte de adultos inescrupulosos, conocedores que, en virtud de ser un grupo de atención prioritaria, reciben un trato a favor en los procesos penales, con sanciones leves enfocadas en su reeducación. Estas consideraciones se sustentan en el principio de proporcionalidad que se sustenta en lo que señala la Constitución de la República (2008) en su artículo 76, numeral sexto, que dice: "La ley establecerá la debida proporcionalidad entre las infracciones y las sanciones penales, administrativas o de otra naturaleza" (p. 53). Claramente se especifica que la aplicabilidad del principio se sustenta en la determinación de las sanciones en congruencia con el daño causado.

El problema entonces surge de la aplicación del principio de proporcionalidad de las penas en los casos de delitos cometidos por menores o adolescentes infractores, por cuanto en delitos calificados como graves y ante la protección que impone el Derecho Constitucional y de Niñez y Adolescencia, parece que existe deficiencia de la finalidad correctiva de la pena. Por lo tanto, el objetivo de esta investigación consiste en analizar y determinar la efectiva aplicación del principio de proporcionalidad de las penas en los delitos cometidos por menores o adolescentes infractores sustentada en las garantías del debido proceso.

\section{DESARROLLO}

\section{El principio de proporcionalidad en el sistema constitucional ecuatoriano}

Doctrinariamente afirma Barnes (1998) que el principio se remonta a la antigüedad y el pensamiento clásico. En su origen como principio de necesidad de la intervención se fue desarrollando en el marco de la jurisprudencia y de la doctrina de Alemania, y abriéndose camino en medio de una confusión terminológica, hasta alcanzar su sentido 
actual. El principio de proporcionalidad de acuerdo con Ferrajoli (1986) hace referencia a que éste se enfoca principalmente en señalar cómo castigar a quien comete un delito. Otra opinión la brinda Bernal (2007), para quien este principio es una guía para aplicar las normas fundamentales desde su institución hasta llegar a la concreción en el proceso. Lopera (2006) por el contrario es más conciso al referir que la proporcionalidad se aplica al sopesar la gravedad tanto de la sanción penal con los hechos delictivos cometidos.

Autores como Villaverde (2008) afirman que este principio asegura la intensidad de la restricción como una sanción impuesta por el Estado, sin que se atente contra los derechos fundamentales o se vulnere el contenido de la norma protegida. Otra definición la brinda Pabón (2007) y a través de ella hace referencia a que se trata de la adecuación efectiva entre la gravedad evidente de la falta con la conducta delictiva asumida por el adolescente. Villaverde (2008) a su vez afirma que este principio asegura la intensidad de la restricción como una sanción impuesta por el Estado, sin que se atente contra los derechos fundamentales o se vulnere el contenido de la norma protegida.

Otra definición importante es la que brinda Aguado (2012), quien menciona que este principio busca obtener el equilibrio entre la vulneración de los bienes y las penas que se van a establecer en búsqueda de la reparación integral, mostrándose como un elemento eficaz y pragmático a la hora de moderar la tensión entre la ciudadanía y el poder político, permitiendo identificar cuatro requisitos que el principio debe cumplir:

a) Definir una finalidad dentro de la legalidad.

b) Encontrarse dentro de los parámetros adecuados e idóneos para hacer efectiva la finalidad legal.

c) Determinar la necesidad de su aplicación, pero salvaguardando a su vez el principio de mínima intervención por parte del poder estatal.

d) Evidenciar la proporcionalidad entre la gravedad de la infracción y el objetivo rehabilitador de la sanción (Arnold, Martínez y Zúñiga, 2012). 
En conjunto estos cuatro requisitos permiten a los estados de derecho, modular no sólo la garantía de los derechos humanos, sino también las libertades del ser humano, sobre todo en aquellas inherentes a la mínima intervención del Estado, en especial en las normas del derecho penal, en donde es evidente la importancia de la relación de proporcionalidad entre la tipicidad y la penalización; también imponiendo a través de éste, el control al poder estatal para que no sobrepase los límites de intervención y aplicación de los derechos fundamentales.

La proporcionalidad de las penas y su aplicación en el Ecuador se sustenta en lo que señala la Constitución de la República (2008) en su artículo 76, numeral sexto, que dice: "La ley establecerá la debida proporcionalidad entre las infracciones y las sanciones penales, administrativas o de otra naturaleza" (p. 53). Por lo tanto se especifica que la aplicabilidad del principio se sustenta en la determinación de las sanciones en congruencia con el daño causado. Señala Yanac (2017) que su aplicabilidad depende del resultado de la ponderación de tres aspectos: 1) la pena debe cumplir con su finalidad de rehabilitación; 2) es evidente que la sanción o pena debe ser aplicada por la necesidad; y, 3) debe existir relación entre la pena y la finalidad de la misma. De esta forma el principio tendrá efectividad en su aplicabilidad.

Los aspectos mencionados deben enmarcarse a su vez en el principio de legalidad, ya que, sin éste, el principio de proporcionalidad se vuelve riesgoso como garantía de los derechos fundamentales constitucionalmente reconocidos. La legalidad, por lo tanto, es el fundamento en el que se sustenta la aplicación proporcional de la pena ante la comprobación de una conducta ilegal. La Constitución de la República (2008) lo determina en su Art. 76, numeral 3: "nadie podrá ser juzgado ni sancionado por un acto u omisión que, al momento de cometerse no esté tipificado en la ley como infracción penal, administrativa u otra naturaleza" (p. 53).

La proporcionalidad es importante para realizar la garantía efectiva de los derechos de las personas, mucho se habla de la proporcionalidad y se tiene claro el concepto y su rol dentro de la figura jurídica constitucional, no obstante, es necesario analizar si en 
realidad se cumple este principio garantista en todo la norma jurídica de un Estado, es por ello que la presente investigación se enfoca en la proporcionalidad constitucional relacionada a las penas que reciben los menores infractores, las mismas que no son proporcionales al daño causado, por cuanto no existe la observancia de los requisitos que tiene que cumplir una pena direccionada a la proporcionalidad del hecho cometido.

\section{El derecho al debido proceso en el juzgamiento de menores infractores}

En la actualidad y tal como lo refiere Donna (2011) la justicia se sustenta en la aplicabilidad de las penas a través de un proceso el cual a su vez da prioridad a lo que son considerados como los derechos y garantías judiciales. Dentro de la misma línea se encuentra la posición de Coke (citado por Donna, 2011), pero hace una interesante referencia a los antecedentes históricos, cuyo origen parece provenir de la Constitución de Inglaterra de 1215, bajo el precepto contenido en el artículo 39 que afirma: "ningún hombre libre puede ser detenido, mantenido preso, expropiado, desterrado, proscrito o de alguna manera destruido, salvo por decisión judicial" (p. 158). En la actualidad los derechos humanos son considerados inviolables y se garantizan dentro de todo Estado de Derechos.

La postura sostenida por Carbonell, Orozco y Vázquez (2002) es que "la adhesión interna a las normas constitucionales y la existencia de una sociedad homogénea, en términos de la igual satisfacción de las necesidades básicas de todos los individuos" ( $p$. 11) de tal forma que todo proceso debe atenerse a la normativa interna y mantener los preceptos constitucionalistas vigentes. La participación del gobierno dentro del sistema judicial de todo Estado debe encaminarse en palabras de estos mismos autores a "prever, con bastante certeza, cómo usará la autoridad, sus poderes coercitivos en determinadas circunstancias y planear los asuntos de los individuos con base en este conocimiento" (p. 15).

Haciendo una breve interpretación generalizada de las definiciones antes mencionadas, se puede afirmar que el debido proceso y sus garantías procesales permiten al ser 
humano obtener respeto a su dignidad humana; por lo tanto, cuando se emiten normas o leyes, estas deben estar fundamentadas en un sistema procesal justo, equitativo y respetuoso de los derechos humanos. Es importante también hacer referencia al afianzamiento de la garantía constitucional a través del control y protección jurisdiccional efectiva que se hace realidad con las acciones que en la Constitución se especifican.

Dentro del Estado de Derechos la figura del juez adquiera relevancia ya que como lo dice Ávila Santamaría (2011) "tiene que aplicar principios que constan en la Constitución y convertirse en cerebro y boca de la Constitución" (p. 135), por lo que la finalidad de mantener este sistema es el "reconocimiento, promoción, garantía de los derechos constitucionalmente establecidos" (p. 146), siendo importante la persona y sus derechos sobre el mismo Estado y las obligaciones. El fundamento y la base es que los derechos están por sobre el Estado, el derecho y cualquier poder, manifestándose en este sentido la misma Constitución de la República (2008) que dice:

"1. El Estado sometido a los derechos:

2. Es deber primordial del Estado garantizar el efectivo goce de los derechos. (Art. 3 , numeral 1)

3. El más alto deber del Estado es respetar y hacer respetar los derechos. (Art. 11, numeral 9)" (Constitución de la República, 2008)

El Estado ecuatoriano se convierte en el garante de los derechos de la ciudadanía, haciendo a su vez que las demás instituciones y colectividad en general respeten estos derechos, en términos de igualdad, no sólo en la parte aplicable a los procedimientos o procesos, sino en todo lo que concierne a la constitucionalidad. Al respecto, Cea (2002) señala que los derechos fundamentales son:

Derechos, libertades, igualdades o inviolabilidades que, desde la concepción, fluyen de la dignidad humana y que son intrínsecos de la naturaleza singular del titular de esa dignidad. Tales atributos, facultades 0 derechos públicos subjetivos son, y deben ser siempre, reconocidos y protegidos por el ordenamiento jurídico, permitiendo al titular exigir su cumplimiento con los deberes correlativos. (p. 265) 
El tratamiento procesal que se les brinda a los menores de edad o adolescentes infractores se guía de acuerdo a lo que se estipula en las Reglas Mínimas de las Naciones Unidas para la Administración de Justicia de Menores o Reglas de Beijing (ONU, 1985), cuyo alcance se fija en que serán aplicadas en los adolescentes que hayan cometido actos delincuenciales, debiendo sus procesos garantizar el cumplimiento de los principios básicos procesales como son la imparcialidad, la no discriminación y el debido proceso en general.

Las mencionadas reglas recomiendan a los Estados parte que emitan disposiciones tendientes a la especificación de la edad penal. De manera concreta estipula que la fijación de la mayoría de edad debe coincidir con el inicio de la "madurez emocional, mental e intelectual" (ONU, 1985, p. 3). Por lo tanto, los adolescentes o menores de edad, no son considerados responsables de sus actos de forma general y de manera concreta, del cometimiento de actos delictivos sino hasta que han alcanzado la mayoría de edad, que en el Ecuador se encuentra señalada en los 18 años conforme lo estipula el Art. 38 del COIP (2014).

\section{La responsabilidad penal de los menores infractores}

A través de los años han sido varios los estudios que se han realizado sobre la delincuencia juvenil, explorando las causas que originan este comportamiento. Se tiene por ejemplo el estudio de Ibáñez (y Graña (2018) quienes han analizado la influencia de la madurez psicosocial de los adolescentes menores de edad en relación con la delincuencia. Los autores han llegado a afirmar que es una realidad la asociación del nivel bajo de madurez con el comportamiento delictivo. Las tendencias a nivel mundial sobre esta problemática señalan que la delincuencia es una forma de comportamiento asociada muy fuerte con la adolescencia y que puede perdurar hasta la adultez temprana (Fariña, Arce y Vázquez, 2011).

Otros autores como Thorberry y otros (2013) señalan que existen tres patrones bien definidos para identificar el aumento de las estadísticas de la delincuencia juvenil: a) un 
aumento de la delincuencia en la adolescencia y una persistencia de altas tasas de criminalidad en la edad adulta, b) la actividad delictiva aumenta en la adolescencia, disminuyendo pronto, durante los años de transición juventud-edad adulta y c) un inicio tardío en el delito al final de la adolescencia y comienzo de la vida adulta.

Las causas por las cuales un adolescente se convierte en un infractor o delincuente son muy diversas: provienen de hogares disfuncionales, los padres se encuentran divorciados y en continúo conflicto, separación familiar por migración, entre otras causas, que por lo general fomentan las conductas antisociales, permiten que el adolescente viva en constante sensación de abandono, se vuelven por lo tanto vulnerables a ser influenciados por personas que pretenden utilizarlos para el cometimiento de delitos.

En consecuencia, los Estados han implementado programas y centros en los cuales los menores de edad sean rehabilitados, lamentablemente las estadísticas anuales no son muy alentadoras ni favorecedoras a las medidas adoptadas, sino que por el contrario, es evidente que cada día la delincuencia y el narcotráfico buscan a los adolescentes menores de edad para que hagan por ellos actos ilegales, convencidos de que si son atrapados por la ley, ésta solo les otorgará medidas leves, que no tienen de proporcional con el daño que ocasionan, en especial cuando los delitos cometidos tienen características de saña, violencia y son graves en las lesiones.

Un punto importante dentro de esta revisión es la imputabilidad. Una definición inicial la brinda Mezger (2005), este autor afirma que "es imputable el que al tiempo de la acción es personalmente exigible para ser imputado a título de culpabilidad" (p. 53). Cabanellas (2010) a su vez menciona que se trata de la capacidad y la aptitud que tiene el ser humano de responder ante la comisión de una falta o delito. Es claro que estas definiciones se sustentan en la exigibilidad, capacidad o aptitud desde la edad que tiene el infractor al momento de cometer un delito.

El tema de la imputabilidad y la responsabilidad penal de los menores de edad, es un tema que genera en la actualidad varias controversias, de manera principal porque los 
legisladores se niegan a reconocer que los adolescentes tienen conciencia del daño que han cometido o están por cometer, lo que es una idea contraria con respecto a la aceptación de que a los 16 años si les conceda el derecho del voto. Es evidente que los legisladores han politizado el tema y prefieren conceder una capacidad de pensamiento madura para elegir en lugar de la responsabilidad para asumir los resultados de sus actos delictivos (Duque, 2016).

Por lo tanto la mayoría de edad y la responsabilidad de los actos por parte de los menores infractores es un punto fundamental para establecer penas y sanciones. En la actualidad está disyuntiva se mantiene, bajo los mismos parámetros: fijar una edad límite en la cual se considere que los niños permanecen sin alcanzar la madurez psicológica y moral como para adquirir responsabilidades ante comportamientos delictivos (Arboleda, Baquero y Domínguez, 2010). En el caso de que no se fije este límite de edad el sentido jurídico de la responsabilidad penal no tendría valor. Por consiguiente, es necesario que se hagan esfuerzos para convenir en una edad mínima razonable que pueda aplicarse a nivel internacional.

En las situaciones de que los adolescentes cometan actos antijurídicos e ilegales, la misma Constitución señala que tienen derecho a un proceso de juzgamiento especial, diferente al de los adultos infractores. La Carta Magna ecuatoriana señala en su artículo 77 , numeral 13 que en los casos de adolescentes infractores las penas se encaminan a medidas socioeducativas que van encauzadas a la rehabilitación sin tener que hacer uso de la privación de la libertad de los menores y sobre todo, que cumpla su pena en centros distintos en los que se encontraran los adultos.

Para algunos autores se trata de un tratamiento lógico, se está ante adolescentes, menores de edad que no están todavía conscientes del alcance de sus actos; sin embargo, para otros, se habla de vulneración de los derechos de las víctimas, enfatizando que el infractor al gozar del beneficio de un proceso de juzgamiento con imposición de penas leves, provocan inseguridad jurídica, por cuanto son pocos los 
adolescentes que no se vuelven reincidentes a causa mismo de la permisividad de la ley vigente.

Hay que poner en relevancia en este punto que en el Ecuador, el Código de la Niñez y Adolescencia (2012) es el cuerpo legal encargado del juzgamiento de adolescentes infractores, lo cual no permite que exista más rigurosidad en la sanción de la infracción, llegando quizá de forma indirecta a que la víctima y familiares de la víctimas, no se sientan satisfechos con la sanción impuesta al adolescente infractor.

Es difícil, pues, que se presente más rigurosidad en la sanción en el Código de la Niñez y Adolescencia, con la simple aplicación de medidas socioeducativas, ya que este código busca la protección de los niños y adolescentes, haciendo que sus derechos se cumplan. No se debe olvidar un punto importante: las víctimas y sus familiares, también son sujetos de derechos, los cuales deben ser respetados; por lo que la ponderación en la equidad de derechos presenta un conflicto, debiendo la ley buscar alternativas para que tanto la víctima como el menor o adolescente infractor, no sientan que sus derechos han sido vulnerados.

De manera general, en el caso de los menores, es inconcebible que este grupo catalogado como de atención vulnerable, pueda ser un instrumento para el cometimiento de delitos graves como son violación, asesinato, robo, etc., debiendo por ello responder ante esta actitud con el cumplimiento de una sanción equiparable con la gravedad del delito cometido. El tema de los menores infractores es un problema que está presente en otros países de América. Así en México, el índice de delitos cometidos por los adolescentes alcanzó un nivel de estadística alarmante, tal como lo señala Aguilar (2018) la actividad delictiva de los menores de edad se está volviendo un negocio aprovechado por sujetos conocedores de las sanciones leves que utilizan a menores para el cometimiento de los mismos.

En Perú, tal como lo señala Pérez (2013) que menciona a la Dirección de Investigación Criminal (Dirincri), el crimen organizado ha involucrado a menores en sus actividades de sicariato, extorsiones, secuestros; conocen además que en caso de ser detenidos 
recibirán sanciones muy bajas, como por ejemplo en el delito de homicidio calificado el tiempo máximo es de seis años en privación de libertad. Las cifras son llamativas, la Tercera Dirección Territorial de la Policía Nacional (Diterpol) ha determinado que aproximadamente un $40 \%$ de los homicidios en Trujillo (Perú) son cometidos por adolescentes cuyas edades varían entre los 14 y 17 años.

La responsabilidad penal de los adolescentes infractores ha sido a su vez un tema de análisis jurídico en algunos países, como por ejemplo en España, el Derecho Penal Juvenil a través de la Ley Orgánica 5/2000 Reguladora de la responsabilidad penal de los menores (LORRPM), ha endurecido las sanciones a menores que llegan a cometer delitos. En este sentido, la política criminal se ha enfocado en dos aspectos: a) incremento de la duración de las sanciones de internamiento; y, b) ampliación de la sanción en régimen cerrado. Se debe hacer énfasis que España es uno de los países que cuenta con disposiciones y normativa jurídica concreta para casos de delincuencia juvenil (Villacreses Briones \& Villacreses Palomeque, 2019).

En el país ibérico se ha reformado la edad en cuanto al régimen cerrado disminuyendo el mínimo de 21 a 18 años, se ha incluido la posibilidad de solicitar medidas cautelares; $y$, el internamiento como una medida cautelar que dura seis meses con la posibilidad de que pueda ser ampliado tres meses adicionales. Aunque se considera que estas medidas equiparan las sanciones para menores con las de adultos y que pueden ser consideradas como contrarias a los intereses de los menores, la jurisprudencia española es clara en enfatizar que cuando se determinan las sanciones penales, éstas no se hacen atendiendo fines particulares, sino aplicar medidas encaminadas a prevención, por ello la proporcionalidad de las penas estipuladas se sustenta en la finalidad que persiguen (García, 2019).

En México la Ley Nacional del Sistema Integral de Justicia Penal para Adolescentes determina que no podrán ser responsables los menores con edades entre los doce años a catorce años, para ello debe comprobarse que el menor haya actuado en obediencia debida tal como lo estipula el Art. 149 de la mencionada Ley; y, que además 
no tenga conocimiento de que ha cometido o va a cometer un acto ilícito con su obediencia. De esta forma los menores no reciben sanciones graves como privación de libertad, sino que los mayores los manejan a sabiendas de que no hay una penalización, por lo que las reincidencias son una constante (Aguilar, 2018).

En Perú el Código Penal en su Art. 20 claramente señala que los menores de 18 años son inimputables, por lo que en caso de cometer algún ilícito la pena que reciben es de apenas seis años en una correccional juvenil. Esta disposición en opinión de Pérez (2013) debe ser reformada ya que la mala conducta de adultos y de menores es considerada como injustificable, debiendo hacer énfasis en que el sistema judicial cumple un rol concreto de administración de justicia (Ibídem). El vecino país de Perú es uno de los que en la región latinoamericana defiende los derechos de los menores de edad, a pesar de que sean utilizados como herramientas delictivas.

\section{METODOLOGÍA}

La modalidad para el desarrollo de la presente investigación fue cualitativa de análisis crítico, ya que se hizo la revisión y se analizó la proporcionalidad de la pena. El tipo de investigación fue explicativa en base al hecho concreto del procesamiento de los menores infractores. Entre los métodos utilizados están: inductivo-deductivo siendo útil este método en la investigación porque se partió de hechos originados dentro del sistema real jurídico y normativo a nivel nacional e internacional; $y$, analítico-sintético utilizado en el desarrollo del trabajo para la reconstrucción de la realidad de cómo se establece la norma jurídica del principio de proporcionalidad. Del mismo modo, por su alcance, la investigación fue exploratoria porque se pretendió familiarizarse con un fenómeno hasta ahora poco estudiado, por cuanto siempre se ha buscado garantizar la proporcionalidad entre el cometimiento de un delito y las penas. La revisión bibliográfica se ha sustentado en el análisis, síntesis y esquematización del marco teórico en base a doctrina, normas jurídicas e internacionales, ubicadas en repositorios jurídicos avalados por la riguridad científica (Peñafiel, Erazo, Pozo y Narváez, 2020). 


\section{APORTES GENERADOS}

El principio de proporcionalidad tiene su origen en la antigüedad; evoluciona dentro del derecho alemán y avanza hasta la era contemporánea siendo considerado como la guía para aplicar las normas fundamentales, aunque manteniendo el equilibrio entre la gravedad del delito y la sanción. Es así como entre la actividad delictiva, el acto ilícito o el delito que se comete hay una relación con el tipo de sanción que debe recibir quien lo ha cometido. En la legislación ecuatoriana está establecido que el principio de proporcionalidad prima en el ámbito penal, administrativo u otra materia; en el cual debe haber congruencia con la gravedad del daño ocasionado, para que así se garantice una sanción justa y legal, con la finalidad de dar rehabilitación a la persona que lo comete y evitar vulneraciones a los derechos humanos.

El principio de legalidad es a su vez la garantía del cumplimiento de la proporcionalidad, teniendo en cuenta que la Constitución de la República del Ecuador hace efectiva mención a que ninguna persona podrá ser llevada a juicio y mucho menos recibir una sanción que no se encuentre determinada o tipificada dentro de la ley correspondiente. Si el principio de proporcionalidad no cumple con el de legalidad, se está frente a una evidente situación de vulnerabilidad de los derechos fundamentales garantizados en la constitución, los que no pueden ser reprimidos ni omitidos por el sistema de justicia estatal, lo que conlleva a una extralimitación del poder punitivo.

El debido proceso son las normas que garantizan el ejercicio de los derechos dentro de la administración pública. La seguridad, la tutela y la protección están vinculados con la legalidad de todo proceso y de forma precisa con la garantía de que al cometimiento de un acto o delito le corresponderá una sanción o pena equivalente a su gravedad. La figura del juzgador cobra relevancia en este punto, ya que le corresponde hacer la valoración de las pruebas para emitir la motivación de su sentencia, en base a normas jurídicas ecuatorianas vigentes.

En el Ecuador el juzgamiento de los adolescentes infractores se encuentra dentro de las disposiciones del Código de la Niñez y Adolescencia; sin embargo, existe una marcada 
desigualdad entre el delito y la sanción, ya que a pesar de que se aplican medidas de privación de libertad, estas son incongruentes con la gravedad de los delitos cometidos. Por lo tanto, se puede afirmar, que en el país el principio de proporcionalidad para las penas en delitos cometidos por adolescentes infractores no es aplicable, evidenciando la falta de rigurosidad del Código de la Niñez y Adolescencia.

La delincuencia juvenil es un problema social con el cual lidian diariamente muchas sociedades. Los delitos en los cuales participan los adolescentes son graves y con características de violencia y saña, que han generado que las estadísticas delictivas aumenten, sobre todo porque al ser un grupo considerado vulnerable por las leyes, se han vuelto instrumentos para delincuentes mayores, quienes usan a los adolescentes para cometer los delitos con la seguridad de que serán juzgados y recibirán una penalidad leve, de poca duración en centros especiales.

Es así que la proporcionalidad de las penas aplicable en los delitos cometidos por menores o adolescentes infractores es un punto de discurso constante para los juristas y la sociedad en general, los primeros porque hacen una ponderación de los derechos inherentes a las partes; los segundos, porque están convencidos que en la actualidad las penas para este grupo humano son irrisorias y no están enfocadas en ser efectivas en la rehabilitación del menor, teniendo en cuenta que las medidas socioeducativas no están dando resultados de disminución de las estadísticas de criminalidad juvenil, sino por el contrario, continúa en la actualidad como un problema social emergente. 


\section{REFERENCIAS CONSULTADAS}

1. Aguado, T. (2012). El principio de proporcionalidad en la jurisprudencia del Tribunal Constitucional peruano. En J. Urquizo, \& N. Salazar, Derecho constitucional Penal. Lima: IDEMSA.

2. Aguilar, P. (septiembre - octubre de 2018). Los menores infractores en la ciudad de México. Revista Jurídica Electrónica Hechos y Derechos(47), s/n.

3. Arboleda, C., Baquero, M., \& Domínguez, M. (enero - diciembre de 2010). La inimputabilidad del menor en el Sistema Penal Colombiano. Universitas Estudiantes(7), 157-174.

4. Arnold, R., Martínez, J., \& Zúñiga, F. (2012). El principio de proporcionalidad en la jurisprudencia del Tribunal Constitucional. Estudios Constitucionales, 10(1), 65-116.

5. Asamblea Nacional. (20 de octubre de 2008). Constitución de la República del Ecuador. Registro Oficial 449 . Quito, Pichincha, Ecuador: Editora Nacional.

6. Asamblea Nacional. (2012). Código Orgánico de la Niñez y Adolescencia. Quito, Pichincha, Ecuador: Corporación de Estudios y Publicaciones.

7. Asamblea Nacional. (10 de febrero de 2014). Código Orgánico Integral Penal. $R$. O. No. 180. Quito, Pichincha, Ecuador: Editora Nacional.

8. Ávila, R. (2011). El neoconstitucionalismo transformador: el Estado y el derecho en la Constitución de 2008. Universidad Politécnica Salesiana. Universidad Andina Simón Bolívar, Sede Ecuador. Fundación Rosa Lusemburg. Quito, Pichincha, Ecuador: Abya Yala. Recuperado el 18 de abril de 2018, de http://hdl.handle.net/10644/2984

9. Barnes, J. (septiembre-diciembre de 1998). El principio de proporcionalidad. Estudio preliminar. Cuadernos de Derecho Público(5), s/p.

10. Bernal, C. (2007). El principio de proporcionalidad y los derechos fundamentales. Bogotá: Centro de Estudios Políticos y Constitucionales.

11. Cabanellas, G. (2010). Diccionario Jurídico de Derecho Usual . Buenos Aires: Heliasta.

12. Calero, A. (12 de septiembre de 2012). Adolescentes infractores, en la mira de Código Penal. Diario El Telégrafo, pág. s/n. Recuperado el 26 de enero de 2020, 
de http://www.telegrafo.com.ec/noticias/judicial/item/adolescentes-infractores-enla-mira-de-codigo-penal.html

13. Carbonell, M. O. (2002). Estado de Derecho. México: Siglo XXI Editores.

14. Carreño, B. (2017). Las penas privativas de otros derechos y sus problemáticas operativas: un análisis a partir de la jurisprudencia colombiana. Trabajo de Grado. Bogotá, Colombia: Universidad Santo Tomás.

15. Cea, J. L. (2002). Derecho Constitucional chileno. Santiago de Chile: Editorial de la Universidad Católica de Chile.

16. Couture, E. (1978). Vocabulario Jurídico. Buenos Aires, Argentina: Depalma.

17. Díaz, A. (2015). Reglas de Beijing, su aplicación en el marco de las leyes 19.970 y 20.084. Revista de Estudios de la Justicia, 19, 207-214. doi:doi:10.5354/07184735.2015.36193

18.Donna, E. (2011). Derecho Penal, Parte General. Buenos Aires: Editorial Rubinzal Culzoni.

19. Duque, L. Z. (2016). La responsabilidad penal de los menores infractores y la edad para la imputabilidad de los adolescentes en delitos contra la vida. Tesis de pregrado. Quito, Pichincha, Ecuador: Universidad de las Américas. Recuperado el 26 de enero de 2020, de http://dspace.udla.edu.ec/handle/33000/5102

20. Fariña, F. A. (2011). Grado de competencia social y comportamientos antisociales, delictivos y no delictivos en adolescentes. Revista Latinoamericana de Psicología, 43(3), 473-486. Recuperado el 26 de enero de 2020, de www.scielo.org.cospdf

21. Fernández, J. (1999). Principios y normas rectoras del derecho penal. Bogotá: Leyer.

22. Ferrajoli, L. (1986). Derecho y razón. Teoría del Garantísmo penal. Madrir: Trotta.

23. García, O. (2019). La contribución de la jurisprudencia al endurecimiento de la respuesta a los menores infractores. Revista Electrónica de Ciencia Penal y Criminología(21), 1-44.

24. Gómez, J. O. (2001). Derecho penal. Parte General (Vol. I). Bogotá: Doctrina y ley. 
25. Ibáñez, V., \& Graña, J. (2018). Madurez psicosocial y comportamiento delictivo en menores infractores. Psicopatología clínica Legal y Forense, 18(1), 1-12. Recuperado el 25 de enero de 2020, de www.dialnet.com

26. Jefatura del Estado. (13 de enero de 2000). Ley Orgánica 5/2000 Reguladora de la Responsabilidad Penal de los Menores . BOE-A-2000-641, No. 11. Madrid, España: Boletín Español.

27. Lopera, G. (2006). Principio de proporcionalidad y Ley penal. Madrid: Centro de Estudios políticos Constitucionales.

28. Mezger, E. (2005). La Culpabilidad. Bogotá: Leyer.

29. ONU. (29 de noviembre de 1985). Reglas Mínimas de las Naciones Unidas para la Administración de la Justicia de Menores o "Reglas de Beijing". Resolución 40/33.

30.Pabón, P. (2007). Comentarios al nuevo sistema de responsabilidad penal paramadolescentes. Bogotá: Ediciones Doctrina y Ley Ltda.

31. Peñafiel Sacoto, J. M., Erazo Álvarez, J. C., Pozo Cabrera, E. E., \& Narváez Zurita, C. I. (2020). La fundamentación y la motivación como habilitantes de la prisión preventiva. Iustitia Socialis, 598.

32. Pérez, M. (2013). Asesinos Adolescentes. Caretas(10), 1-10.

33. Ruíz, C. E. (2006). Teoría de los fines de la pena (Segunda ed.). Bogotá: U. Externado de Colombia.

34. Thornberry, T. G. (2013). Transición desde la delincuencia juvenil a la delincuencia adulta. Revista Española de Investigación Criminológica, 11(2), s/n.

35. Vargas, L. (2010). Las penas y medidas de seguridad consecuencia del Derecho Punitivo en México. Letras Jurídicas(10), 1-10.

36. Villaverde, I. (2008). La resolución de conflictos entre derechos fundamentales. El principio de proporcionalidad. En M. Carbonell, El principio de proporcionalidad y la interpretación constitucional. Quito: Ministerio de Justicia y Derechos Humanos.

37. Villacreses Briones, T., \& Villacreses Palomeque, J. (2019). La constitucionalización del principio de proporcionalidad en España y la actividad legislativa penal. IUSTITIA SOCIALIS, 4(1), 4-15. doi:http://dx.doi.org/10.35381/racii.v4i1.535 
38. Yanac, J. (2017). El delito de estafa y el principio de proporcionalidad de la pena en el Código Penal peruano vigente. Grado Académico. Lima, Perú: Universidad Inca Garcilaso de la Vega. Recuperado el 26 de enero de 2020, de http://repositorio.uigv.edu.pe/handle/20.500.11818/2119

(C2020 por los autores. Este artículo es de acceso abierto y distribuido según los términos y condiciones de la licencia Creative Commons Atribución-NoComercial-Compartirlgual 4.0 Internacional (CC BY-NC-SA 4.0) (https://creativecommons.org/licenses/by-nc-sa/4.0/). 